\title{
Cats match voice and face: cross- modal representation of humans in cats (Felis catus)
}

\section{$\operatorname{AUTHOR}(\mathrm{S})$ :}

Takagi, Saho; Arahori, Minori; Chijiiwa, Hitomi; Saito, Atsuko; Kuroshima, Hika; Fujita, Kazuo

\section{CITATION:}

Takagi, Saho ...[et al]. Cats match voice and face: cross-modal representation of humans in cats (Felis catus). Animal cognition 2019, 22(5): 901-906

\section{ISSUE DATE:}

2019-09

URL:

http://hdl.handle.net/2433/243873

\section{RIGHT:}

This is a post-peer-review, pre-copyedit version of an article published in 'Animal cognition'. The final authenticated version is available online at: https://doi.org/10.1007/s10071-019-01265-2.; The full-text file will be made open to the public on 10 May 2020 in accordance with publisher's 'Terms and Conditions for Self-Archiving; This is not the published version. Please cite only the published version.; この論文は出版社版でありません。引用の際には出版社版をご確認ご 利用ください。 
1 Cats match voice and face: cross-modal representation of humans in cats (Felis catus)

2 Saho Takagi ${ }^{1,3}$

3 Minori Arahori ${ }^{1,3}$

4 Hitomi Chijiiwa ${ }^{1,3}$

$5 \quad$ Atsuko Saito ${ }^{2}$

6 Hika Kuroshima ${ }^{1}$

$7 \quad$ Kazuo Fujita ${ }^{1}$

8

9 1Affiliation: Department of Psychology, Graduate School of Letters, Kyoto

10 University

11 'Affiliation address: Yoshida-honmachi, Sakyo, Kyoto, 606-8501, Japan.

12 2 Affiliation: Department of Psychology, Faculty of Human Sciences, Sophia

13 University

142 Affiliation address: 7-1, Kioicho, Chiyoda-ku, Tokyo, 102-8554, Japan

15 3Affiliation: Japan Society for the Promotion of Science

16 3Affiliation address: 5-3-1, Chiyoda-ku, Tokyo, 102-0083, Japan.

18 Corresponding author: Saho Takagi, takagi.saho.67x@st.kyoto-u.ac.jp 
21 Abstract

22 We examined whether cats have a cross-modal representation of humans, using a cross-modal expectancy violation paradigm originally used with dogs by Adachi et

24 al. (2007). We compared cats living in houses and in cat cafés to assess the potential effect of postnatal experience. Cats were presented with the face of either their owner or a stranger on a laptop monitor after playing back the voice of one of two people calling the subject's name. In half of the trials the voice and face were of the same person (congruent condition) whereas in the other half of trials the stimuli did not match (incongruent condition). The café cats paid attention to the monitor longer in incongruent than congruent conditions, showing an expectancy violation. By contrast, house cats showed no similar tendency. These results show that at least café cats can predict their owner's face upon hearing the owner's voice, suggesting possession of cross-modal representation of at least one human. There may be a minimal kind or amount of postnatal experiences that lead to formation of a cross-modal representation of a specific person.

36 Keywords: Cross-modal representation, Cats, Felis catus, Expectancy violation method 


\section{Introduction}

$$
\text { Integration of multi - sensory information facilitates the detection or }
$$

identification of external stimuli. For example, often we hear someone's voice

calling us, but we cannot see the person. In this situation we can recall the person's face. This shows that we have a mental representation that integrates information from visual and auditory modalities (cross-modal representation) (see Campanella and Belin 2007 for review). In humans this ability emerges early in life. Bahrick et al. (2005) reported that even 4- to 6-month-old infants perceived face - voice relations of unfamiliar adults.

Nonhuman animals also have cross-modal representation of others. This should be an important ability especially for social animals living in complex societies; allowing them to identify individuals, avoid conflicts and maintain social balance, rank, and perhaps cooperation. Some social species are known to have cross-modal representations of conspecifics (chimpanzees (Pan troglodytes): Kojima et al. 2003, rhesus macaques (Macaca mulatta): Adachi and Hampton 2011; Sliwa et al. 2011, Grey-Cheeked mangabeys (Lophocebus albigena): Bovet and Deputte 2009, horses (Equus caballus): Proops et al. 2009, lions (Panthera leo): Gilfillan et al. 2016, goats (Capra hircus): Pitcher et al. 2017, crows (Corvus macrorhynchos): Kondo et al. 2012). Furthermore, rhesus monkeys, squirrel monkeys (Saimiri 
57 boliviensis) and dogs (Canis familiaris) can also form cross-modal representation of

58 familiar members of at least one other species, namely humans (Adachi et al. 2007;

$59 \quad$ Adachi and Fujita 2007; Sliwa et al. 2011).

Adachi and Fujita (2007) reported that squirrel monkeys responded differently

depending on the familiarity of the human stimuli, using a symbolic matching-to-

sample task. They trained monkeys to match photographs of two caretakers and a

symbolic visual stimulus. One caretaker was a primary caretaker, more familiar

than the other (secondary caretaker). In test trials, a voice which belonged to either

the primary or secondary caretaker was played back immediately after the visual

sample stimulus disappeared, then two comparison stimuli appeared, one of which

the monkey was required to choose. The authors predicted that congruency

between voice and sample stimulus would affect matching accuracies. Results

showed that accuracies did not differ between congruent and incongruent trials

when the primary caretaker's face was the sample, but accuracies were higher in

congruent than incongruent trials when the secondary caretaker's face was the

sample. Thus, the secondary caretaker's voice did not interfere with matching the

primary caretaker's face to the symbolic stimulus, whereas the primary caretaker's 
symbolic stimulus. Thus, familiarity of the specific person affected the monkeys' cross-modal representation.

Adachi et al. (2007) reported that pet dogs have a cross-modal representation of their owner. Dogs were presented with a photo of either their owner's or a stranger's face on a monitor after a voice calling subject's name was played back. The voice and face matched in half of the trials and mismatched in the other half. Results showed that dogs looked at the photo longer in both incongruent conditions, suggesting that they predicted the owner's face upon hearing the owner's voice, and another face upon hearing a stranger's voice. Conceivably, extensive experience with a specific person strengthens the formation of such cross- $^{-}$ modal representations. Do other companion animals show the same tendency as dogs?

Like dogs, cats are a popular companion animal for humans, and recent studies have shown that like dogs, cats also have remarkable social cognitive abilities. They respond to human pointing cues (Miklósi et al. 2005) and gaze cues (Pongrácz et al. 2018), discriminate human emotional expressions (Galvan and Vonk 2016) and human attentional states (Ito et al. 2016), and refer to human facial expressions in the presence of a mildly frightening object (Merola et al. 
93 2015).Saito and Shinozuka (2013), using a habituation-dishabituation procedure,

94 reported that cats discriminated their owner's voice from a stranger's voice.

95 However, it is unknown whether they predict their owner's face after hearing the

96 owner's voice, as expected if integration of the relevant audio-visual information

$97 \quad$ occurs.

98 Here we asked whether cats (Felis catus) have cross-modal representations of

99 their owners, using the task originally used with dogs in Adachi et al. (2007). If

100 familiarity of the person affects cross-modal representation, as seen in previous

101 studies, the rearing environment should affect formation of a cross-modal

102 representation of the owner. More specifically, house cats - with a closer

103 relationship with their owner - should show stronger results than cats living at a

104 cat café where many people interact with them each day. In previous research data

105 from these two groups of cats analyzed separately their responses to human voices

106 were different (Saito et al. 2019). The expectancy violation-based prediction was

107 that if cats have a cross-modal representation of their owner they should pay attention to the monitor for longer in incongruent (mis-matching) conditions than congruent (matching) conditions. 
112 Subjects

113 Eighty-seven domestic cats (Felis catus) (48 males, 39 females) participated. Forty-

114 three were kept at five "cat cafés" (24 males, 19 females, mean age 4.14 years, $S D=$

1152.98 years, range 4 months to 10.7 years), where many unfamiliar visitors have

116 contact with the cats. There are various types of cat cafes in Japan. Some serve both

117 as a normal cat café where visitors can enjoy interacting with cats and consider

118 fostering a cat. Cats leave these cafés when they find a foster family. We tested cats

119 in cafes where the cats were permanent residents and where they spend all their

120 time. The remaining subjects were house cats (24 males, 20 females, mean age 5.14

121 years, $S D=3.18$ years, range 8 months to 12.4 years). Subjects had been with their owner for at least for 4 months in cat cafés and 11 months in households. An additional 23 cats (12 cats from cat cafés and 11 from households) were excluded due trials) (17). In addition to approval from the institutional animal experiment committee (see paragraph on compliance with ethical standards), informed consent study. 
131 The auditory stimuli consisted of a recording of either the owner or a same-sex

132 unfamiliar person (stranger) calling the subject's name once. Each owner was

133 instructed to call out the cat's name as they normally would; the stranger was

134 instructed to call out the name in the way the owner did. We recorded the calls

135 using a handheld digital audio recorder (Roland EDIROL R-09, Japan) in WAV

136 format. The sampling rate was $44,100 \mathrm{~Hz}$ and the sampling resolution was 16-bit.

137 We used 1-s call stimuli regardless of the cats' names; all voices were adjusted to

138 the same volume with the help of version 2.3.0 of Audacity $(\mathrm{R})$ recording and editing

139 software (Audacity Team 2018). Voice stimuli were played from a speaker (Sanwa

140 MM-SPS2UBK, Japan) connected to a laptop personal computer (NEC Lavie G

141 type Z, Japan) which controlled all experimental stimuli. The visual stimuli

142 consisted of a photo of the face of either the owner or a stranger. We took a digital,

143 full-face, color photo of each person smiling, and stored the photo in PNG format.

144 Presented photos were ca. $16.5 \times 16 \mathrm{~cm}$ on the 13.3 -in. monitor of the laptop computer. The background was always black.

HDR-CX390, Japan; SONY HDR-CX675, Japan), one placed in front of subject, 
148 another placed slightly to one side, and the other placed behind subject; all

149 cameras focused on the cat.

151 Procedure

152 Cats were tested individually in their familiar place: house or café. Before testing

153 we waited until cats appeared relaxed in the presence of the experimenter; this

154 took about 15 min for house cats whereas almost all café cats ended no such

155 familiarization time. An experimenter gently restrained the cat on the floor in front

156 of the laptop computer, about $40 \mathrm{~cm}$ away. The experimenter started a trial by

157 pressing a key on the computer when the subject was looking toward the monitor.

158 Each trial consisted of two phases: the voice phase and the face phase. In the voice

159 phase, one stimulus voice was played back from the speakers linked to the laptop

every $1 \mathrm{~s}$, for a total of four presentations. Immediately after the fourth auditory

161

stimulus either the owner's or a stranger's face appeared on the monitor for 7-s

162

(face phase) (see Fig.1). The experimenter restrained the cat throughout the voice

phase and released it at the start of the face phase; some cats stayed, whereas monitor disappeared. 
and voice: owner-congruent, owner-incongruent, stranger-congruent, and stranger-

incongruent. For example, in the owner-incongruent condition, a stranger's voice

was played in the voice phase, but the owner's face appeared in the face phase.

These four trials were presented in pseudo-random order with the restriction that

the same voice was never repeated on consecutive trials. received four trials in a single session, with an inter-trial interval of at least 3 min. be placed in front of the monitor; three subjects participated in only the first trial, three in the first and second trials, while four subjects received no fourth trial.

During the interval, cats acted freely in the experimental room. The experimenter restraining the cat was ignorant of the condition; she closed her eyes during the test trials and avoided making eye contact with the subject. Presentation of voice and face stimuli was controlled via a Visual Studio 2013 program on the laptop personal computer. 
184 Analysis

185 A coder (H.C.), blind to the conditions, counted the number of frames (30

186 frames/1 sec.) in which cats paid attention to the monitor in the face phase (total 7

187 sec) for each condition. Paying attention was defined as looking at or sniffing the

188 monitor. We could not discriminate these two acts because a few cats did not touch

189 the monitor with their nose while sniffing. Trials in which subject did not look at

190 the monitor at all were excluded from the analyses because we could not know if

191 expectancy violation occurred. Sixty-four trials were excluded for café cats, 65 for

192 house cats (no significant difference; Fisher's Exact Test: $p=.73$ ). Table 1 shows

193 valid data points, i.e., the number of trials cats looked at the monitor in each

194 condition. The videos were analyzed using Adobe Premiere CS6 (USA) software.

195 To check the reliability of coding, an assistant who was blind to the conditions

196 coded a randomly chosen $20 \%$ of the videos. The correlation between the two coders

197 was excellent for time spent paying attention to the monitor (Pearson's $r=0.97, n$

$198=40, p<0.01)$.

199 All statistical analyses were conducted with $\mathrm{R}$ version 3.5.1 (R Core Team, 
202 2015), in which face (owner/stranger), congruency (congruent/incongruent), home

203 environment (café/house) and an interaction between congruency and home

204 environment were entered as fixed factors and subject identity was entered as a

205 random factor. To test whether effects of factor were significant, we ran $\mathrm{F}$ tests by

206 an Anova function in car package (Fox et al 2012). We used a difflsmeans function

207 in lmerTest package (Kuznetsova Brockhoff and Christensen 2017) which tested

208 differences of least squares means to compare each condition. Degrees of freedom

209 were adjusted by Kenward-Roger and p-value was adjusted by the Holm procedure.

210

211 Results

212 Fig. 2 shows time spent paying attention to the monitor during the face phase

213 in café cats (A) and house cats (B). Contrary to our prediction, café cats showed

214 more attention to the monitor in both incongruent conditions, whereas house cats

215 showed no clear tendency; they attended to the monitor almost randomly. LMM

216 revealed that significant main effects of congruency $(F(1,60.87)=4.10, p=.04)$,

217 home environment $(F(1,79.03)=8.06, p<.01)$, and an interaction between

218 congruency and home environment $(F(1,60.71)=7.76, p<.01)$. There was no

219 significant main effect of face $(F(1,96.78)=0.06, p=.79)$. 
221 between congruent and incongruent conditions in café cats $(p<.01)$, between café

222 cats and house cats in congruent conditions $(p<.01)$, and between café cats in

223 congruent conditions and house cats in incongruent conditions $(p<.01)$.

224

225

Discussion

226

We used an expectancy violation procedure to ask whether cats have a cross-

227

modal representation of their owner. We presented the face of either the owner or

228

stranger after playing back the voice of the owner or a stranger calling the subjects'

229

name. Results showed that café cats paid attention to the monitor for longer in

230

both incongruent conditions, when voices and face were mismatched, whereas

231

house cats showed no clear trends. These results contradict our prediction and

232

suggest clearly that café cats predict the owner's face upon hearing the

233

corresponding voice, demonstrating a cross-modal representation of a specific

234 person; whether house cats have this cross-modal ability remains to be further

235 examined. The results also indicate that cross-modal representations of others are

236 not exclusive to species that form complex social groups, such as dogs, but also

237 more solitary species, such as cats (Bradshaw 2016).

238 There are three possible explanations for our failure to demonstrate a cross-

modal representation in house cats. First, cross-modal representation of a specific 
240 person might be affected by factors other than familiarity with that individual.

241 Café cats typically see and interact with multiple strangers on a daily basis. People

242 with greater experience of heterospecific faces can discriminate them better than

243 people with fewer such experiences (Dufour and Petit 2010). Also, older captive

244 chimpanzees discriminated human faces better than younger chimpanzees

245 probably because older chimpanzees had more experiences to see a variety of

246 human faces (Dahl et al 2013). These results raise the possibility that café cats

247 greater experience of a variety of human faces and voices might result in better

248 discrimination abilities.

249 Second, greater experience of seeing and interacting with people might promote

250 application of an "exclusive rule." Our task required cats to predict a stranger from

251 a stranger's voice in one of the incongruent conditions. It should be more difficult to

252 predict the stranger from the stranger's voice than the owner from the owner's

253 voice using and exclusion rule. Kondo et al (2012) demonstrated that crows did not

254 react even when a familiar crow's calls were played back followed by an unfamiliar

255 bird presented visually, suggesting that they did not exclusively predict "a

256 stranger". Similar asymmetrical results were obtained in horses (Proops and

257 McComb 2012). In contrast, café cats showed expectancy violation in both 
258 incongruent conditions; they showed no asymmetry. This suggests that the café

259 cats exclusively predicted a non-owner face, using the exclusion rule. Conceivably,

260 increased opportunities to see various people might improve cross-modal

261 representations of others. Home environments that differ from normal pet

262 environments in terms of seeing human strangers might explain why café cats

263 showed the clearer expectancy violation.

264 Finally, house cats might have been more nervous during the test. We asked the

265 owner to remain in another room because we wanted to test cats' representation of

266 their owner. Some cats may not have felt sufficiently at ease in the presence of only

267 the experimenter. More house cats remained immobile after the experimenter

268 released them in the face phase; a freezing reaction might have resulted in longer

269 looking times in all conditions compared to café cats. To exclude such a possibility

270 future work should use a more natural experimental setting less likely to cause stress in house cats, or conduct a test that objectively estimates their stress level.

272 One may argue that house cats did not discriminate between the owner's face and a stranger's face, given their lack of differential responses across conditions.

274 However, previous studies have shown that house cats respond differently to 
276 Thompson Guijarro and Zulch 2015; Galvan and Vonk 2016). Further research

277 should be conducted on cats' ability to discriminate the owner's face from a

278 stranger's face when only visual information is presented.

279 We used voice and face to examine cats' cross-modal recognition of humans.

280 However, cats also use their olfactory sense to recognize others (Gorman and

281 Trowbridge 1989). Further research should examine whether olfactory information

282 is also integrated in cross-modal representations of others.

283 Cross-modal recognition is not limited to a one-to-one relation (owner's voice -

284 face) as in this study. For example, dogs can show more general cross-modal

285 recognition: Taylor Reby and McComb (2011) examined whether dogs could match

286 frequency of growls and dogs' body size. Dogs spent more time looking at a correct

287 model (small body - high frequency, or big body - low frequency) than an incorrect

288 model (small body - low frequency, or big body - high frequency), suggesting that

289 they relate information about body size to "voices." Furthermore, dog cross-modally

290 matched a human male or female voice and a male or female face (Takaoka et al

291 2013). It is still unknown whether cats have similar cross-modal recognition

292 abilities beyond one-to-one correspondence; this is another issue for future study. 
300 thanks all owners and cats who volunteered in this study. The authors also wish to

Kyoto University. 
311

312

\section{Reference}

Adachi, I., \& Fujita, K. (2007). Cross-modal representation of human caretakers in squirrel monkeys. Behavioural Processes, 74, 27-32.

Adachi, I., \& Hampton, R. R. (2011). Rhesus monkeys see who they hear: spontaneous cross-modal memory for familiar conspecifics. PLoS One, 6, $\mathrm{e} 23345$.

Adachi, I., Kuwahata, H., \& Fujita, K. (2007). Dogs recall their owner's face upon hearing the owner's voice. Animal Cognition, 10, 17-21.

Audacity Team (2018). Audacity(R): Free Audio Editor and Recorder [Computer application]. Version 2.3.0 retrieved December 20th 2018 from https://audacityteam.org/ .

Bahrick, L. E., Hernandez-Reif, M., \& Flom, R. (2005). The development of infant learning about specific face-voice relations. Developmental Psychology, 41, $541-552$.

Bates, D., Maechler, M., Bolker, B., \& Walker, S. (2015). Fitting Linear MixedEffects Models Using lme4. Journal of Statistical Software, 67, 1-48.

Bovet, D., \& Deputte, B. L. (2009). Matching vocalizations to faces of familiar conspecifics in grey-cheeked mangabeys (Lophocebus albigena). Folia Primatologica, 80, 220-232. 
330 Bradshaw, J. W. (2016). Sociality in cats: A comparative review. Journal of

Campanella, S., \& Belin, P. (2007). Integrating face and voice in person perception.

Collard, R. R. (1967). Fear of strangers and play behavior in kittens with varied social experience. Child Development, 877-891.

Dahl, C. D., Rasch, M. J., Tomonaga, M., \& Adachi, I. (2013). Developmental processes in face perception. Scientific Reports, 3, 1044.

Ellis, S. L. H., Thompson, H., Guijarro, C., \& Zulch, H. E. (2015). The influence of body region, handler familiarity and order of region handled on the domestic cat's response to being stroked. Applied Animal Behaviour Science, 173, 60-67.

Fox, J., Weisberg, S., Adler, D., Bates, D., Baud-Bovy, G., Ellison, S., \& Heiberger, R. (2012). Package 'car'. Vienna: R Foundation for Statistical Computing.

Galvan, M., \& Vonk, J. (2016). Man's other best friend: domestic cats (F. silvestris catus) and their discrimination of human emotion cues. Animal Cognition, 19, 193-205. recognition in wild African lions. Biology Letters, 12, 20160323. 
348 Gorman, M. L., \& Trowbridge, B. J. (1989). The role of odor in the social lives of

349 carnivores. In Carnivore behavior, ecology, and evolution (pp. 57-88). Springer,

$350 \quad$ Boston, MA.

351 Ito, Y., Watanabe, A., Takagi, S., Arahori, M., \& Saito, A. (2016). Cats beg for food

352 from the human who looks at and calls to them: Ability to understand humans'

$353 \quad$ attentional states. Psychologia, 59, 112-120.

354 Kojima, S., Izumi, A., \& Ceugniet, M. (2003). Identification of vocalizers by pant

355 hoots, pant grunts and screams in a chimpanzee. Primates, 44, 225-230.

356 Kondo, N., Izawa, E. I., \& Watanabe, S. (2012). Crows cross-modally recognize

357 group members but not non-group members. Proceedings of the Royal Society of London B: Biological Sciences, 279, 1937-1942.

359 Kuznetsova, A., Brockhoff, P. B., \& Christensen, R. H. B. (2017). lmerTest package: tests in linear mixed effects models. Journal of Statistical Software, 82.

Merola, I., Lazzaroni, M., Marshall-Pescini, S., \& Prato-Previde, E. (2015). Social referencing and cat-human communication. Animal Cognition, 18, 639-648. study of the use of visual communicative signals in interactions between dogs 
(Canis familiaris) and humans and cats (Felis catus) and humans. Journal of

Pitcher, B. J., Briefer, E. F., Baciadonna, L., \& McElligott, A. G. (2017). Cross$4,160346$.

Pongrácz, P., Szapu, J. S., \& Faragó, T. (2018). Cats (Felis silvestris catus) read human gaze for referential information. Intelligence, in press.

Proops, L., \& McComb, K. (2012). Cross-modal individual recognition in domestic horses (Equus caballus) extends to familiar humans. Proceedings of the Royal Society of London B: Biological Sciences, rspb20120626.

Proops, L., McComb, K., \& Reby, D. (2009). Cross-modal individual recognition in domestic horses (Equus caballus). Proceedings of the National Academy of Sciences, 106, 947-951.

R Core Team (2018). R: A language and environment for statistical computing. $R$ Foundation for Statistical Computing, Vienna, Austria. URL https://www.R-

380 project.org/. 
383 Saito, A., Shinozuka, K., Ito, Y., \& Hasegawa, T. (2019) Domestic cats (Felis catus)

384 discriminate their names from other words, Scientific Reports, in press.

385 Sliwa, J., Duhamel, J. R., Pascalis, O., \& Wirth, S. (2011). Spontaneous voice-face

386 identity matching by rhesus monkeys for familiar conspecifics and humans.

387 Proceedings of the National Academy of Sciences, 108, 1735-1740.

Takagi, S., Arahori, M., Chijiiwa, H., Tsuzuki, M., Hataji, Y., \& Fujita, K. (2016). There's no ball without noise: cats' prediction of an object from noise. Animal Cognition, 19, 1043-1047.

Takaoka, A., Morisaki, A., \& Fujita, K. (2013). [in Japanese with English abstract] Cross-modal concept of human gender in dogs (Canis familiaris). The Japanese Journal of Animal Psychology, 63, 123-130. 
$398 \quad$ Legend

$399 \quad$ Fig. 1

400 Fig.1 Diagram illustrating each condition. Face was presented in the monitor (Face

401 phase) immediately after voices was played back (Voice phase). The face and voice

402 matched in half of the trials (congruent condition) whereas they mismatched in the

403 other half of trials (incongruent condition). Black line represents Congruent

404 conditions, dotted line represents Incongruent conditions.

407 Time spent paying attention to the monitor in (A) Café cats and (B) House cats in the Face phase. White bar represents congruent conditions, Black bar represents

409 incongruent conditions. Error bar indicates SE. Unit of Y axis is frames ( 30 frames/1

410 sec.).

413 Table. 1 The number of valid data points representing the number of trials cats

414 looked at the monitor in each condition. 
Figure 1

\section{Voice phase}

Face phase

Owner voice Stranger voice

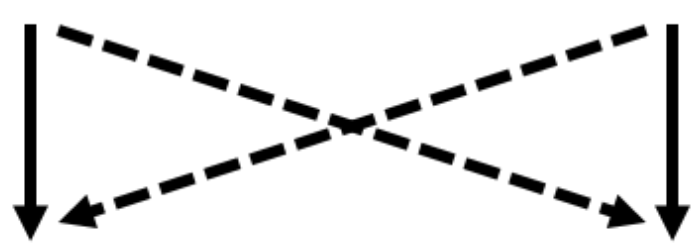

Owner face

Stranger face

$\longrightarrow$ Congruent condition
$\longrightarrow \rightarrow$ Incongruent condition 
Figure 2
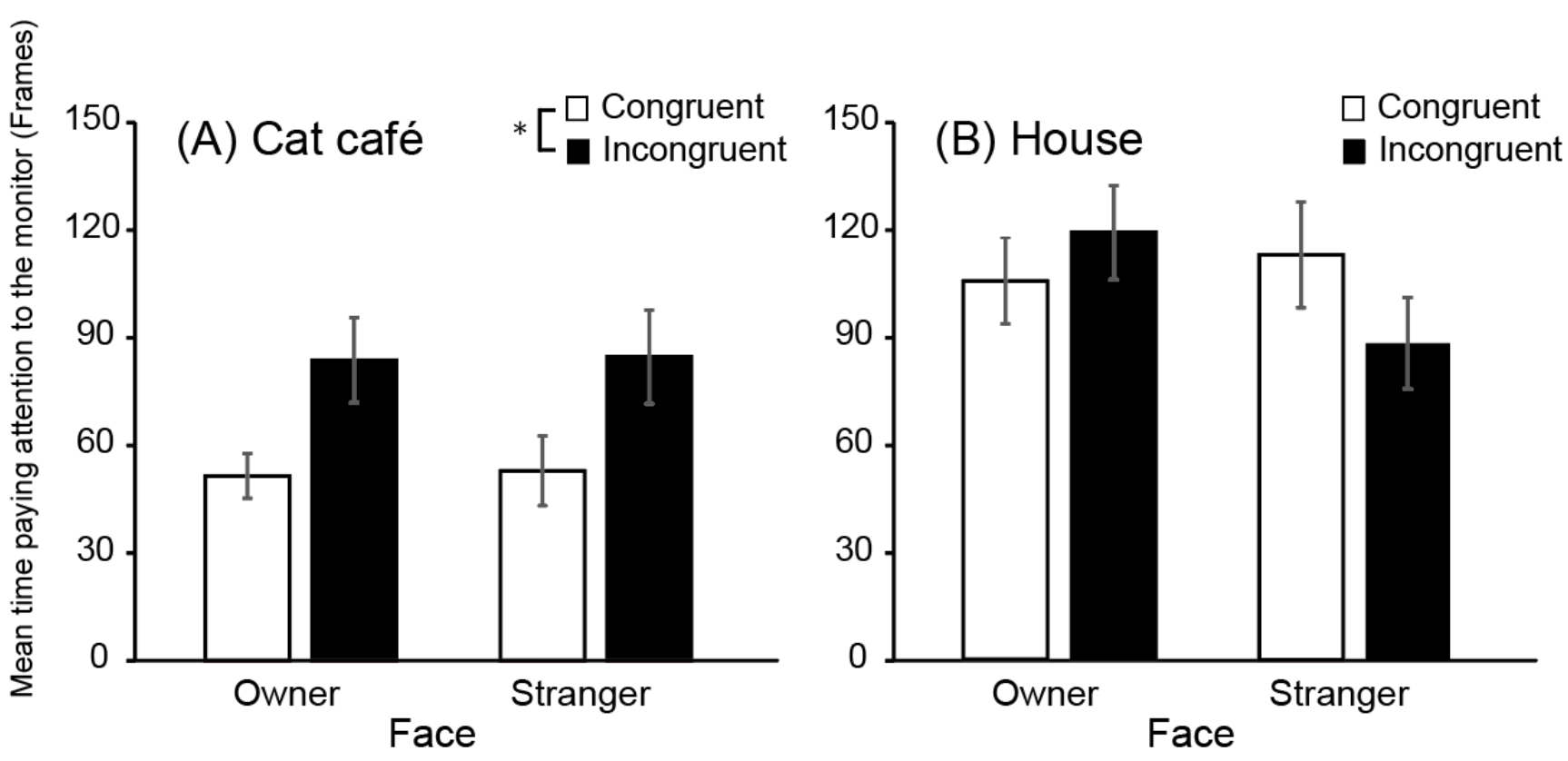


\section{Table. 1}

\section{Face Owner Stranger}

\section{Congruent Incongruent Congruent Incongruent}

Café

22

28

23

22

House

29

23

28

25 